\title{
the humangenome
} Everyone's genome

"The human genome underlies the fundamental unity of all members of the human family, as well as the recognition of their inherent dignity and diversity. In a symbolic sense, it is the heritage of humanity." versal Declaration on the Human Genome and Human Rights (http://wrvww_unesco_org/human_rights/hrbc.htm) strike a personal chord with many people. And application of this knowledge will, in time, materially benefit almost everyone in the world.

It is with particular pleasure, therefore, that we present this special section of Nature on the human genome. It provides a comprehensive overview of current knowledge, in three sections:

The News and Views articles provide the context, communicate the excitement, and present a critical evaluation of the papers.

The Analysis section delves into the opportunities offered to biologists by the genome and explores the approaches and tools needed to exploit these opportunities.

- The Research section comprises a set of papers that, for the most part, report the findings of the international consortia of scientists that make up the Human Genome Project. More than 2,500 authors from 20 laboratories contributed to these papers.

Recognizing the need for engaging educational material, the issue is accompanied by a wall chart, "The Geography of the Genome", and an educational CD-ROM produced by the US National Human Genome Research Institute (and co-sponsored by Nature). It includes a historical timeline of genetics, the background to the Human Genome Project, and teaching aids to explain the fundamental principles of genetics.

A suite of interwoven maps is one of the features of the issue. The key map for the entire project is the whole-genome clone-based physical map (p. 934) constructed by the International Human Genome Mapping Consortium. It provided the scaffold upon which the sequence was assembled. The cytogenetic map, described on p. 953, plots landmarks across the genome and anchors the physical map to the underlying chromosomal positions. A comparison of the genetic and physical maps (p. 951) charts the rate of recombination — or exchange between each pair of our 46 chromosomes - that occurs as the genome is passed on through generations. Finally, the International SNP [single nucleotide polymorphism] Map Working Group documents 1.42 million polymorphic sites in the genome (p. 928), providing for the first time a variant in virtually every gene and in each genomic region. This map of human variation will facilitate efforts to reconstruct our evolutionary history and dissect the genetic basis of human traits and disease.

Analysis of the draft genome sequence itself (p. 860) provides the first panoramic view of the landscape of our genome. We learn the extent to which it has been colonized by parasitic DNA elements. In times past, these elements underwent massive proliferation, playing an important role in shaping the evolution of the human genome. Another notable feature is the much lower gene tally than anticipated, which indicates that human complexity does not arise solely from the number of genes.

Although computational predictions can provide 'best guesses' on gene number and structure, definitive answers require experimental data on gene expression, in terms of both temporal and positional analyses of gene products. As a step towards this, microarray technology has been used to validate gene predictions and more accurately define gene structures (p. 922).

One principle at the heart of the Human Genome Project, reflected in the Universal Declaration on the Human Genome and Human Rights, is free and unlimited access to the sequence. In keeping with this principle, the entire content of this section, plus additional features and commentary, is available without restriction (http://www.nature.com/genomics).

We welcome feedback on any aspect of this publication by e-mail at genome@nature.com.

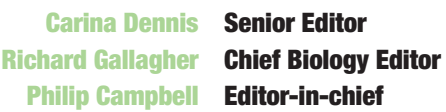

